\title{
Cosmology with self-interacting sterile neutrinos and dark matter: A pseudoscalar model
}

\author{
Maria Archidiacono, ${ }^{1}$ Steen Hannestad, ${ }^{1,2}$ Rasmus Sloth Hansen, ${ }^{1,3}$ and Thomas Tram ${ }^{4}$ \\ ${ }^{1}$ Department of Physics and Astronomy, Aarhus University, 8000 Aarhus C, Denmark \\ ${ }^{2}$ Aarhus Institute of Advanced Studies, Aarhus University, 8000 Aarhus C, Denmark \\ ${ }^{3}$ School of Physics, The University of New South Wales, Sydney, NSW 2052, Australia \\ ${ }^{4}$ Institut de Théorie des Phénomènes Physiques, École Polytechnique Fédérale de Lausanne, \\ CH-1015 Lausanne, Switzerland
}

(Received 30 April 2014; revised manuscript received 20 January 2015; published 18 March 2015)

\begin{abstract}
Short baseline neutrino oscillation experiments have shown hints of the existence of additional sterile neutrinos in the eV mass range. Such sterile neutrinos are incompatible with cosmology because they suppress structure formation unless they can be prevented from thermalizing in the early Universe or removed by subsequent decay or annihilation. Here, we present a novel scenario in which both sterile neutrinos and dark matter are coupled to a new, light pseudoscalar. This can prevent thermalization of sterile neutrinos and make dark matter sufficiently self-interacting to have an impact on galactic dynamics and possibly resolve some of the known problems with the standard cold dark matter scenario. Even more importantly it leads to a strongly self-interacting plasma of sterile neutrinos and pseudoscalars at late times and provides an excellent fit to cosmic microwave background data. The usual cosmological neutrino mass problem is avoided by sterile neutrino annihilation to pseudoscalars. The preferred value of $H_{0}$ is substantially higher than in standard $\Lambda$ CDM (lambda cold dark matter) and in much better agreement with local measurements.
\end{abstract}

DOI: 10.1103/PhysRevD.91.065021

PACS numbers: 14.60.St, 14.60.Pq, 98.80.Cq, 98.80.Es

\section{INTRODUCTION}

Data from a number of neutrino oscillation experiments point to the existence of a fourth, sterile neutrino with a mass around $1 \mathrm{eV}$ (see e.g. Refs. [1,2]). However, such a neutrino would be completely thermalized in the early Universe through a combination of mixing and scattering [3-5], and since there are stringent cosmological constraints on the presence of eV-scale neutrinos [6,7], cosmology seems at odds with the oscillation experiments unless the sterile neutrino is somehow prevented from being fully thermalized in the early Universe [8] (see also Refs. [9-11]).

Several simple solutions exist to this problem. First of all, it is entirely possible that the underlying cosmological model differs from the standard $\Lambda \mathrm{CDM}$ universe, and in more complex models, constraints on light neutrinos can be severely weakened. Even if $\Lambda \mathrm{CDM}$ does turn out to be the correct cosmological model, oscillation data can still be made compatible provided that the sterile neutrino is at most partly thermalized or is removed by decay and/or annihilation before the rest mass becomes important for cosmological structure formation.

The generic condition for producing any given particle species is that $\Gamma>H$ at some epoch, where $\Gamma$ is the production rate and $H$ is the expansion rate of the Universe. Partial thermalization can be achieved either by lowering $\Gamma$ or by increasing $H$. Models which lower $\Gamma$ are for example models with new interactions in the sterile sector [12-15], whereas $H$ can be modified for example in models with low reheating temperature or early dark energy [16].
In this paper we will revisit the possibility of new interactions in the sterile sector. Previous studies have all focused on interactions via a new light vector, i.e. a Fermilike interaction [12-15]. This has the merit of making neutrinos strongly interacting at early times while completely decoupled at late times. Here, we will investigate a new possibility-coupling neutrinos (and possibly dark matter) to a massless or very light pseudoscalar such as the majoron. Couplings to a scalar would lead to the presence of a new fifth force on which very tight bounds exist. However, since the pseudoscalar couples only to the spin of the involved particles and because macroscopic media are unpolarized, no such problem exists for pseudoscalars.

Interactions via a light pseudoscalar have the interesting property that they make the sterile neutrinos very strongly self-interacting at late times and effectively remove sterile neutrino anisotropic stress. Depending on the density of sterile neutrinos, this property could allow us to distinguish between self-interacting and free-streaming sterile neutrinos.

If dark matter couples to the same particle, it has the possibility to make the scattering cross section strongly velocity dependent through Sommerfeld enhancement which is a desirable feature if some of the astrophysics problems related to cold dark matter are to be addressed.

\section{MODEL FRAMEWORK}

Instead of constructing an explicit model, we base our discussion on a simplified setup which, however, does contain all the relevant physics. The sterile neutrino is 
coupled to a new light pseudoscalar with mass $m_{\phi} \ll 1 \mathrm{eV}$ via

$$
\mathcal{L} \sim g_{s} \phi \bar{\nu} \gamma_{5} \nu .
$$

Later we will look at dark matter with a similar coupling to $\phi$,

$$
\mathcal{L} \sim g_{d} \phi \bar{\chi} \gamma_{5} \chi
$$

One important note is in order at this point: We assume the coupling to be diagonal in mass basis, such that the three mainly active mass states are completely uncoupled. This is the most natural assumption given that $\phi$ is associated with new physics and not related to standard model flavor. The new interaction is also felt partly by the active Standard Model neutrinos, although suppressed by the mixing angle. Limits from cosmology [17] are not relevant, as the active neutrino mass states do not feel the new coupling, but constraints from supernovae $[18,19]$ and laboratory measurements [20] do apply. The supernova bounds are derived by requiring that the pseudoscalars do not carry away a significant amount of the energy released by the supernova which results in a bound on the coupling of electron neutrinos to the pseudoscalar [18], $g_{e} \lesssim 4 \times 10^{-7}$. If the coupling becomes much larger, the pseudoscalars will be caught in the supernova, and the bound disappears again. However, almost all of these values are excluded by laboratory experiments [20], and we will only consider the supernova limit here. For the sterile neutrinos, the bound on $g_{e}$ comes from the process $\nu_{e} \nu_{e} \rightarrow \phi$, and it translates into the bound $g_{s} \lesssim g_{e} / \sin ^{2} \theta_{s}=3 \times 10^{-5}$, using $\sin ^{2} 2 \theta_{s} \sim 0.05$ from the short baseline experiments [1,2], where $\theta_{s}$ is a mixing angle representative for $\left(\nu_{e}, \nu_{s}\right)$ mixing or $\left(\nu_{\mu}, \nu_{s}\right)$ mixing. Although supernovae give the strongest bounds on the coupling strength, they are quite dependent on details in the assumptions about the supernova, and it might be more appropriate to quote the bound as $g_{s} \lesssim 10^{-4}$.

Let us now go through the implications of this new interaction, first for the sterile neutrinos and subsequently for the dark matter.

\section{STERILE NEUTRINOS}

The new interaction introduces a matter potential for sterile neutrinos of the form [21,22]

$$
V_{s}\left(p_{s}\right)=\frac{g_{s}^{2}}{8 \pi^{2} p_{s}} \int p d p\left(f_{\phi}+f_{s}\right)
$$

where $f_{\phi}$ is the Bose-Einstein distribution for the pseudoscalar and $f_{s}$ is the distribution for the sterile neutrinos (see e.g. Refs. [3,23-26] for a discussion of matter potentials in the standard model). Note that the potential in Eq. (3) arises from bubble diagrams and is nonzero even in a $C P$-symmetric medium.

Before proceeding with a quantitative calculation, we can estimate how large $g_{s}$ needs to be in order to block thermalization. Consider a scenario with thermal $\phi$ and $\nu_{s}$ distributions characterized by a common temperature $T$. The potential is then

$$
V_{s} \sim 10^{-1} g_{s}^{2} T .
$$

In the absence of nonstandard effects, the sterile neutrinos would be thermalized through oscillations at $T \sim$ $10\left(\delta m^{2} / \mathrm{eV}^{2}\right)^{1 / 6} \mathrm{MeV} \sim 10 \mathrm{MeV}$ [3]. To prevent this effect, we need to suppress the mixing angle in matter, $\theta_{m}$, as the production rate is proportional to $\sin ^{2} 2 \theta_{m}$. This is achieved if the matter potential dominates the energy difference associated with vacuum oscillation, i.e.

$$
V \gtrsim \frac{\delta m_{\nu_{s}}^{2}}{2 E} \sim \frac{\delta m_{\nu_{s}}^{2}}{T},
$$

prior to neutrino decoupling at $T \sim 1 \mathrm{MeV}$ so that

$$
g_{s}^{2} \gtrsim 10 \frac{\delta m_{\nu_{s}}^{2}}{T^{2}} \sim 10^{-11} .
$$

So a priori we expect that a value of $g_{s} \sim 3 \times 10^{-6}$ is sufficient to block thermalization. It should be noted here that since the pseudoscalar coupling is diagonal in the mass basis the active state feels an additional matter potential associated with the $\phi$ background. The magnitude of the potential felt by the active state is approximately $V \sim \sin ^{2}\left(\theta_{s}\right) V_{s} \sim 0.01 V_{s}$. The only effect is a minute shift in the effective mass difference, corresponding to a shift of less than $1 \%$ in $g_{s}$.

\section{THERMAL HISTORY OF THE STERILE NEUTRINO}

The sterile neutrino can in principle be thermalized via incoherent processes such as $\phi \phi \leftrightarrow \bar{\nu}_{s} \nu_{s}$, assuming that there is a preexisting background of $\phi$. The thermally averaged cross section in the highly relativistic limit can be calculated to be [27]

$$
\langle\sigma|v|\rangle=\frac{g_{s}^{4}}{8 \pi T^{2}} .
$$

Conservatively assuming that $g_{s} \sim 10^{-4}$, we find that $\nu_{s}$ and $\phi$ come into equilibrium at a temperature of $T \sim 1 \mathrm{GeV}$, i.e.

\footnotetext{
${ }^{1}$ For active-active oscillations, this additional potential is important, but effects from active-active oscillations are expected to be small as all active neutrinos have almost identical spectra in the early Universe.
} 
significantly before the oscillation process becomes important [28]. However, since the dark sector is decoupled, it does not share the entropy transfer to the standard model particles, and the end result is that when oscillations become important at $T \sim 10 \mathrm{MeV}$ a low-temperature background of $\phi$ and $\nu_{s}$ exists. However, if $g_{s}$ is significantly lower, no thermalization occurs before the oscillation period.

\section{RESULTS AND NUMERICAL IMPLEMENTATION}

We compute the thermalization process by solving the quantum kinetic equations (QKEs) for a simplified twoneutrino framework with oscillations between $\nu_{\mu}$ and $\nu_{s}$ using a modified version of our public code LASAGNA [29]. The formulation of the QKEs [3,4,23-25,30,31] is based on an expansion of the density matrices, $\rho$, in terms of $P_{a}, P_{s}$, $P_{x}$, and $P_{y}$,

$$
\rho=\frac{1}{2} f_{0}\left(\begin{array}{cc}
P_{a} & P_{x}-i P_{y} \\
P_{x}+i P_{y} & P_{s}
\end{array}\right),
$$

where $f_{0}$ is the Fermi-Dirac distribution function. The QKEs are now

$$
\begin{aligned}
& \dot{P}_{a}=V_{x} P_{y}+\Gamma_{a}\left[2-P_{a}\right], \\
& \dot{P}_{s}=-V_{x} P_{y}+\Gamma_{s}\left[2 \frac{f_{\mathrm{eq}, s}\left(T_{\nu_{s}}, \mu_{\nu_{s}}\right)}{f_{0}}-P_{s}\right], \\
& \dot{P}_{x}=-V_{z} P_{y}-D P_{x}, \\
& \dot{P}_{y}=V_{z} P_{x}-\frac{1}{2} V_{x}\left(P_{a}-P_{s}\right)-D P_{y} .
\end{aligned}
$$

Here, the potentials are given by

$$
\begin{aligned}
& V_{x}=\frac{\delta m_{\nu_{s}}^{2}}{2 p} \sin 2 \theta_{s}, \\
& V_{z}=-\frac{\delta m_{\nu_{s}}^{2}}{2 p} \cos 2 \theta_{s}-\frac{14 \pi^{2}}{45 \sqrt{2}} p \frac{G_{F}}{M_{Z}^{2}} T^{4} n_{\nu_{s}}+V_{s},
\end{aligned}
$$

where $p$ is the momentum, $G_{F}$ is the Fermi coupling constant, $M_{Z}$ is the mass of the $Z$ boson, and $n_{\nu_{s}}=$ $\int f_{s} d^{3} p /(2 \pi)^{3}$ is the number density of sterile neutrinos. For the repopulation of the active neutrinos, we use the expression

$$
\Gamma_{a}=C_{\mu} G_{F}^{2} p T^{4}, \quad C_{\mu} \approx 0.92 .
$$

For the sterile neutrino redistribution, we choose $T_{\nu_{s}}$ and $\mu_{\nu_{s}}$ to conserve energy and number density, when $f_{\text {eq }, s}=\left(e^{p / T_{\nu_{s}}-\mu_{\nu_{s}} / T_{\nu_{s}}}+1\right)^{-1}$, and we approximate the rate by

$$
\Gamma_{s}=\frac{g_{s}^{4}}{4 \pi T_{\nu_{s}}^{2}} n_{\nu_{s}} .
$$

Finally, we approximate the damping term by $D=\frac{1}{2}\left(\Gamma_{a}+\Gamma_{s}\right)$.

We compute the sterile neutrino contribution to the potential in Eq. (3) from the actual numerical distribution. The contribution from the $\phi$-background is computed analytically assuming that the $\phi$-particles were produced thermally above a TeV. They will then follow a BoseEinstein distribution with a reduced temperature of

$$
T_{\phi}=\left(\frac{g_{\star}\left(T_{\gamma}\right)}{g_{\star}(1 \mathrm{TeV})}\right)^{\frac{1}{3}} T_{\gamma} \simeq\left(\frac{10.75}{106.7}\right)^{\frac{1}{3}} T_{\gamma} \simeq 0.47 T_{\gamma},
$$

where the approximation is valid in the temperature range of interest. We are ignoring momentum transfer between the sterile neutrinos and the pseudoscalars for simplicity, but we suspect that including it would have a negligible effect on our results. When sterile neutrinos are produced, they will create nonthermal distortions in the sterile neutrino distribution, and the sterile neutrino spectrum might end up being somewhat nonthermal. In Fig. 1 we show the final contribution to the energy density $N_{\text {eff }}$,

$$
N_{\text {eff }} \equiv \frac{\rho_{\nu_{a}}+\rho_{\nu_{s}}}{\rho_{\nu_{0}}}, \quad \text { where } \rho_{\nu_{0}} \equiv \frac{7}{8}\left(\frac{4}{11}\right)^{4 / 3} \rho_{\gamma},
$$

from a sterile neutrino with mixing parameter $\sin ^{2} 2 \theta_{s}=$ 0.05 and $m_{\nu_{s}}=1 \mathrm{eV}$, close to the best fit value from neutrino oscillation data [1,2]. The transition from full thermalization to zero thermalization happens in the

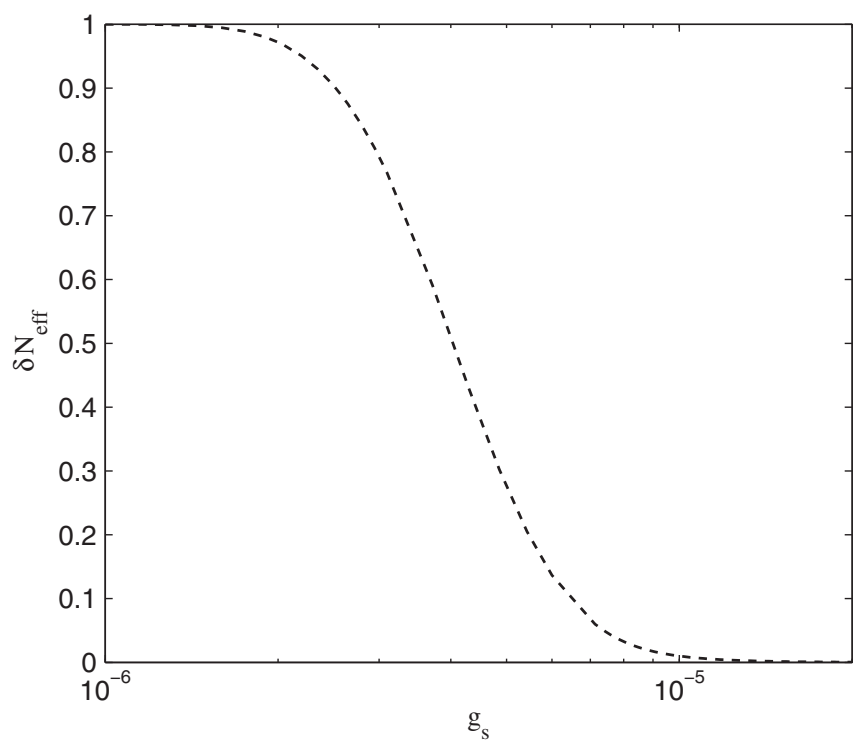

FIG. 1. The contribution of the sterile neutrino to the relativistic energy density $\delta N_{\text {eff }}=N_{\text {eff }}-3$ as a function of the coupling parameter $g_{s}$. 
region $10^{-6}<g_{s}<10^{-5}$, confirming the simple estimate in Eq. (6). ${ }^{2}$

\section{LATE-TIME PHENOMENOLOGY}

In a recent paper by Mirizzi et al. [32] it was pointed out that even if strong self-interactions prevent thermalization of the sterile neutrino before active neutrino decoupling the active and the sterile neutrino species will eventually be almost equilibrated by oscillations at late times. This leads to a scenario in which active and sterile neutrino distributions have similar temperatures and both contribute to the combined $N_{\text {eff }}$. Even if early thermalization is prevented, this still leads to a sterile neutrino population with a temperature only slightly below that of standard model active neutrinos, and therefore the usual cosmological neutrino mass bound still applies to this model.

However, unlike the previously studied Fermi-like interaction, sterile neutrinos and pseudoscalars interact via a variety of $2 \leftrightarrow 2$ processes which in general have a scattering rate of order $\Gamma \sim g_{s}^{4} T$ because there is no mass scale involved. This is true for example for the pair annihilation process $\nu_{s} \bar{\nu}_{s} \rightarrow \phi \phi$ where we already found the thermally averaged cross section to be $\langle\sigma|v|\rangle=g_{s}^{4} /\left(8 \pi T^{2}\right)$ in the relativistic limit, implying a reaction rate $\Gamma=\langle\sigma|v|\rangle n_{\nu_{s}} \approx$ $3.6 \times 10^{-3} g_{s}^{4} T$. This should be compared to the Hubble expansion rate $H \sim 10 T^{2} / m_{\mathrm{Pl}}$. As long as $g_{s} \gtrsim 10^{-6}$, the $\nu_{s}-\phi$ plasma becomes strongly self-interacting before the sterile neutrinos become nonrelativistic around recombination. Therefore, the rest mass constraint does not apply to this model; as soon as sterile neutrinos become nonrelativistic, they annihilate into $\phi$. This annihilation has two immediate effects. It leads to an overall increase in the energy density of the $\nu_{s}-\phi$ fluid, and it leads to a temporary decrease in the equation of state parameter for the fluid. Both of these effects were discussed in detail in Ref. [33].

The strong self-interactions of the combined fluid also leads to a complete absence of free-streaming and in turn an absence of anisotropic stress in the $\nu_{s}-\phi$ plasma. The scenario where all neutrinos are strongly interacting is strongly disfavoured by current data (see e.g. [17,33-40] for discussions of self-interacting neutrinos and cosmic structure formation). However, this is not necessarily true for models in which standard model neutrinos are free streaming, and the interaction is confined to the sterile sector. We note here that since the pseudoscalar coupling is diagonal in mass basis it does not induce self-interactions in the three active mass states.

We have performed a study of how this model is constrained by current cosmic microwave background

\footnotetext{
${ }^{2}$ Note that in the absence of a preexisting population of $\phi$ and $\nu_{s}$, sterile neutrino production would still be suppressed for the same values of $g_{s}$ as soon as a small amount of $\nu_{s}$ has been produced through oscillations. The assumption is thus not crucial to the scenario.
}

(CMB) data through a Markov chain Monte Carlo (MCMC) sampling of the cosmological parameter space performed with CosmoMC [41] and using CMB data from the Planck mission as well as CMB polarization data from the WMAP satellite [42] (we refer to this data combination as "Planck + WP"). We describe the neutrino sector by the overall energy density after thermalization, $N_{\text {eff }}$, and assume a sterile mass of $1 \mathrm{eV}$. We assume that all neutrino species and the pseudoscalar equilibrate at some temperature between the thermalization scale at a few $\mathrm{MeV}$ and the CMB scale $(T \sim 1 \mathrm{eV})$, so that the energy density in the active sector is $21 / 32 N_{\text {eff }}$ with the remaining $11 / 32 N_{\text {eff }}$ in the $\nu_{s}-\phi$ fluid.

In the top panel of Fig. 2 we show the one-dimensional marginalized posterior for $N_{\text {eff }}$ for the Planck + WP data,
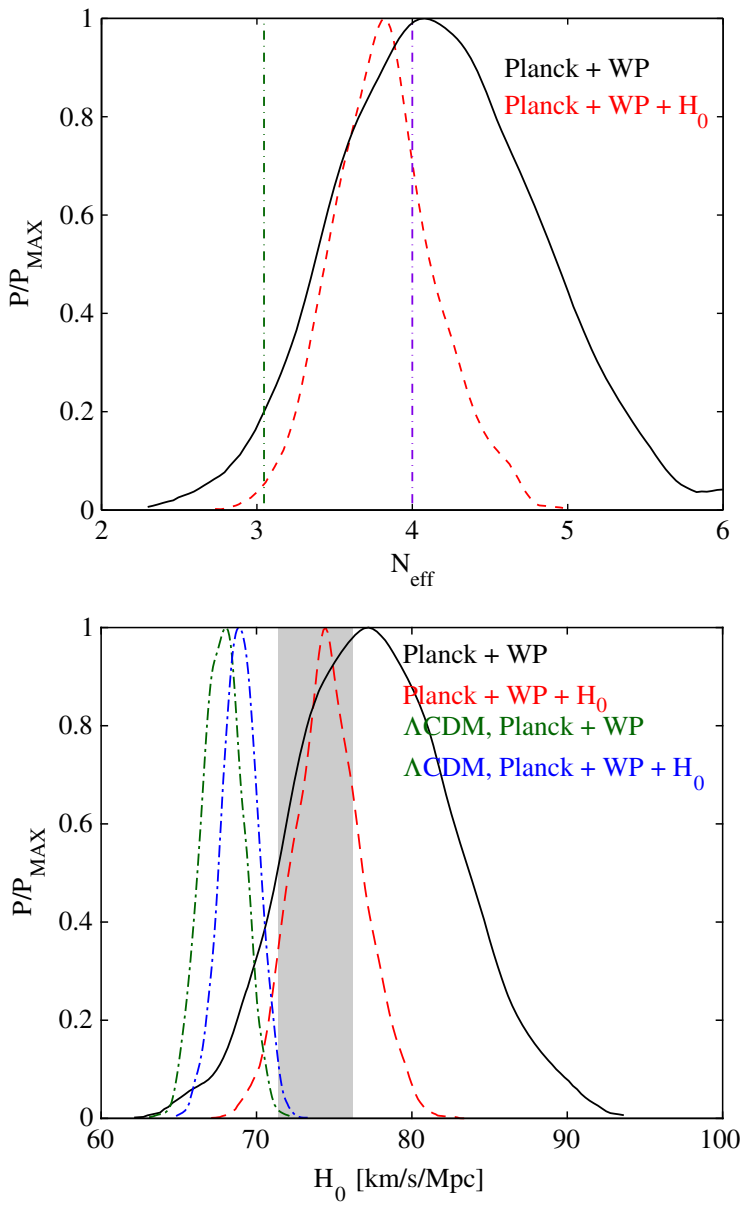

FIG. 2 (color online). One-dimensional marginalized posteriors for $N_{\text {eff }}$ (top panel) and $H_{0}$ (bottom panel) obtained by assuming the pseudoscalar scenario and using only CMB data (black/solid line) and $\mathrm{CMB}$ data plus the $H_{0}$ prior (red/dashed line). (Top panel) The green dash-dot line refers to the $\Lambda$ CDM model $\left(N_{\text {eff }}=3.046\right)$, and the purple line is the complete thermalization case $\left(N_{\text {eff }} \simeq 4\right)$. (Bottom panel) The green and the blue dash-dot lines show the posteriors obtained in the $\Lambda \mathrm{CDM}$ model using Planck and Planck $+H_{0}$, respectively. The $H_{0}$ prior is marked by the gray shaded region [43]. 
as well as for the same data, but with the direct measurement of $H_{0}$ from Ref. [43] included. The data show a clear preference for high values of $N_{\text {eff }}$, and the most extreme case with complete thermalization of the sterile neutrino, corresponding to $N_{\text {eff }} \simeq 4$, is well within the $1 \sigma$ allowed region. It is also of interest to compare the difference in $\chi^{2}$ between this model and the standard $\Lambda \mathrm{CDM}$ cosmology. We find that $\Delta \chi^{2}$ of the pseudoscalar model compared to the reference $\Lambda \mathrm{CDM}$ model is $\Delta \chi^{2}=\chi_{\text {pseudoscalar }}^{2}-\chi_{\Lambda \mathrm{CDM}}^{2}=1.3$, while if in our model we assume $N_{\text {eff }} \simeq 4$, then $\Delta \chi^{2}=0.6$.

Interestingly for this model with a subdominant, strongly interacting neutrino sector, we also find a preference for a higher value of $H_{0}$. This effect was seen already in Ref. [33] but with a much more dramatic increase in $H_{0}$ because all neutrinos were assumed to be strongly interacting. In the bottom panel of Fig. 2, we show the onedimensional marginalized posterior for $H_{0}$ for this model as well as for $\Lambda \mathrm{CDM}$. The increase in $H_{0}$ alleviates the tension between the locally measured value of $H_{0}$ and the much lower value inferred from Planck data when the standard model is assumed. We see this effect very directly when comparing $\chi^{2}$ values, $\Delta \chi^{2}=\chi_{\text {pseudoscalar }}^{2}-\chi_{\Lambda \mathrm{CDM}}^{2}=-2.5$, while if in our model we assume $N_{\text {eff }} \simeq 4$, then $\Delta \chi^{2}=-3.9$. We thus find that in this case the model with a strongly interacting $\nu_{s}-\phi$ sector is a better fit to current data than $\Lambda C D M$ (and of course a vastly better fit than $\Lambda \mathrm{CDM}$ with an additional $1 \mathrm{eV}$ sterile neutrino).

\section{DARK MATTER}

We will now investigate the possibility that dark matter also couples to the new pseudoscalar with a dimensionless coupling strength, $g_{d}$. We assume that the dark matter is produced at a very high temperature by e.g. inflaton decay. Once dark matter is coupled to the new interaction, there is the potential worry that it will pair annihilate via the process $\chi \bar{\chi} \rightarrow \phi \phi$ with the same cross section as in Eq. (7). If the annihilation process is in equilibrium where $\chi$ goes nonrelativistic, it will dilute the density of $\chi$ while transferring an unacceptable amount of entropy to $\phi$. Due to the nature of the interaction, it is decoupled at high temperatures, and the cross section likewise drops when the dark matter becomes nonrelativistic. Therefore, we only need to ensure that the dark matter annihilation rate is low enough at $T_{\max } \sim m_{\chi}$. We assume that the cross section is given by the highly relativistic expression for $\langle\sigma|v|\rangle$ in Eq. (7) and use the condition $\Gamma\left(T_{\max }\right)=\langle\sigma|v|\rangle n_{\chi}<H\left(T_{\max }\right)$ to derive the condition,

$$
g_{d} \lesssim 2 \times 10^{-5}\left(\frac{m_{\chi}}{\mathrm{MeV}}\right)^{1 / 4},
$$

for the new interaction not to overly dilute the density of $\chi$.

Additionally, the new coupling also induces a Yukawatype potential between the dark matter particles. This in turn leads to dark matter self-interactions which might have observable consequences for galactic dynamics. Rather than going through a detailed calculation, we will simply estimate the mean time between dark matter scatterings in order to estimate whether self-interactions are important. To do so we will follow the prescription given in Ref. [44]. First, following Ref. [45] we write

$$
V(r)=-\frac{g_{d}^{2}}{m_{\chi}^{2}} \frac{e^{-m_{\phi} r}}{4 \pi r^{3}} h\left(m_{\phi} r\right) \mathcal{S},
$$

where $h\left(m_{\phi}, r\right)=1+m_{\phi} r+\frac{1}{3}\left(m_{\phi} r\right)^{2}$ and $\mathcal{S}$ is a spindependent factor which we assume to be 1 .

The interaction potential in Eq. (11) causes elastic scattering of dark matter, and following the prescription in Ref. [44], we can estimate the value of $g_{d}$ needed in order to have a significant impact on galactic dynamics. The calculation in Ref. [44] was performed for a massless $U(1)$ vector, so the potential is Coulomb-like. This in turn leads to both "soft" and "hard" scattering of roughly equal importance. Here, we can safely neglect the contribution from soft scatterings because of the steepness of the potential.

The ratio of the scattering time scale $\tau_{\text {scat }}$. to the dynamical time scale in the galaxy $\tau_{\text {dyn }}$ is given by Eq. (17) in Ref. [44],

$$
\frac{\tau_{\mathrm{scat}}}{\tau_{\mathrm{dyn}}}=\frac{2 R^{2}}{3 N \sigma},
$$

where $R$ is the radius of the galaxy, $N$ is the number of dark matter (DM) particles in the galaxy, and $\sigma$ is the scattering cross section. For a hard scatter, we have $\sigma \simeq b^{2}$ where the impact parameter $b$ is the radial distance such that the sum of kinetic and potential energy is zero,

$$
\frac{\alpha_{d}}{m_{\chi}^{2} b^{3}}=\frac{1}{2} m_{\chi} v^{2}
$$

where we have used that $m_{\phi} b \sim m_{\phi} / m_{\chi} \ll 1$ which leads to the approximation $V(r) \approx-\alpha_{d} /\left(m_{\chi}^{2} r^{3}\right)$ where $\alpha_{d}=g_{d}^{2} / 4 \pi$. We then find that

$$
\left(\frac{\tau_{\mathrm{scat}}}{\tau_{\mathrm{dyn}}}\right)^{3}=\frac{2 R^{4} m_{\chi}^{8} G^{2}}{27 N \alpha_{d}^{2}}
$$

where $G$ is Newton's constant. The condition for the time scale of scattering to be less than the age of the Universe is ${ }^{3}$ $\tau_{\text {scat }} / \tau_{\text {dyn }} \lesssim 50$. Plugging in numbers for a Milky Way-size halo and using $\alpha_{d}=g_{d}^{2} / 4 \pi$, we find

\footnotetext{
${ }^{3}$ We take $\tau_{\text {dyn }}$. to be the dynamical time scale of a Milky Waysize halo.
} 


$$
g_{d} \gtrsim 6 \times 10^{-8}\left(\frac{m_{\chi}}{\mathrm{MeV}}\right)^{\frac{9}{4}} .
$$

The value of $g_{d}$ in Eq. (15) can be seen as a lower bound on the value required to have a significant effect. The actual value required might be somewhat larger.

For elastic scattering to be important in itself, the mass of the dark matter particle is therefore required to be quite small. For example, $g_{d} \sim 10^{-5}$ leads to the requirement that $m_{\chi} \lesssim 10 \mathrm{MeV}$. So depending on the unknown mass of the dark matter particle, hard scattering on this potential could have a direct impact on galactic dynamics. Even if this is not the case, the potential could still have a very important indirect effect through the Sommerfeld mechanism [45]. The idea is that the dark matter particles could have some weak short-range scattering cross section generated by beyond the standard model (BSM) physics, which is then enhanced by a velocity-dependent boost factor $S(v)$ such that $\sigma(v)=S(v) \sigma_{0}$. If this new BSM physics enters at a scale $\Lambda_{\mathrm{BSM}}$, we could expect $\sigma_{0} \sim 1 / \Lambda_{\mathrm{BSM}}^{2}$.

\section{SOMMERFELD ENHANCED SCATTERING}

The potential in Eq. (11) diverges faster than $r^{-2}$, so it is singular and leads to an unbounded Hamilton operator [46]. This is of course not physical, since the potential will ultimately be regularized by UV physics. While the boost factor can be made independent of the regularization procedure, it will depend a bit on the UV completion $[45,46]$. We are just trying to estimate this effect, so we follow the simplified version of the regularization procedure outlined in Ref. [45]: We introduce a cutoff in the potential defined by $V\left(r_{\text {cut }}\right)=\Lambda_{\mathrm{BSM}}$ and set $V\left(r<r_{\text {cut }}\right) \equiv$ $V\left(r_{\text {cut }}\right)$ such that the potential is continuous at $r_{\text {cut }}$.

To compute the Sommerfeld factor, we follow Ref. [45] and write the radial part of the Schrödinger equation as

$$
\begin{aligned}
\Phi_{\ell}^{\prime \prime}(x) & =\left(\frac{m_{\chi}}{p^{2}} V\left(\frac{x}{p}\right)+\frac{\ell(\ell+1)}{x^{2}}-1\right) \Phi_{\ell}(x), \\
& =\left(\frac{-g_{d}^{2} v}{8 \pi x_{m}^{3}} h\left(F x_{m}\right) e^{-F x_{m}}+\frac{\ell(\ell+1)}{x^{2}}-1\right) \Phi_{\ell}(x),
\end{aligned}
$$

with $x \equiv p r$ and $F \equiv \frac{2 m_{\phi}}{m_{v} v}$. The continuous box renormalization has been implemented by simply using $x_{m} \equiv$ $\max \left(x, x_{\text {cut }}\right)$ inside the potential term. The equation determining the cutoff $x_{\text {cut }}$ is

$$
1=\left(\frac{m_{\chi}}{\Lambda_{\mathrm{BSM}}}\right) \frac{g_{d}^{2} v^{3}}{32 \pi x_{\mathrm{cut}}^{3}} h\left(F x_{\mathrm{cut}}\right) e^{-F x_{\mathrm{cut}}}
$$

In the limit $x \rightarrow 0$, the complete solution to Eq. (16) is $A x^{\ell+1}+B x^{-\ell}$ for $\ell \geq 0$. As usual, requiring the solution to be regular at $x=0$ forces $B=0$. $A$ can be absorbed into the
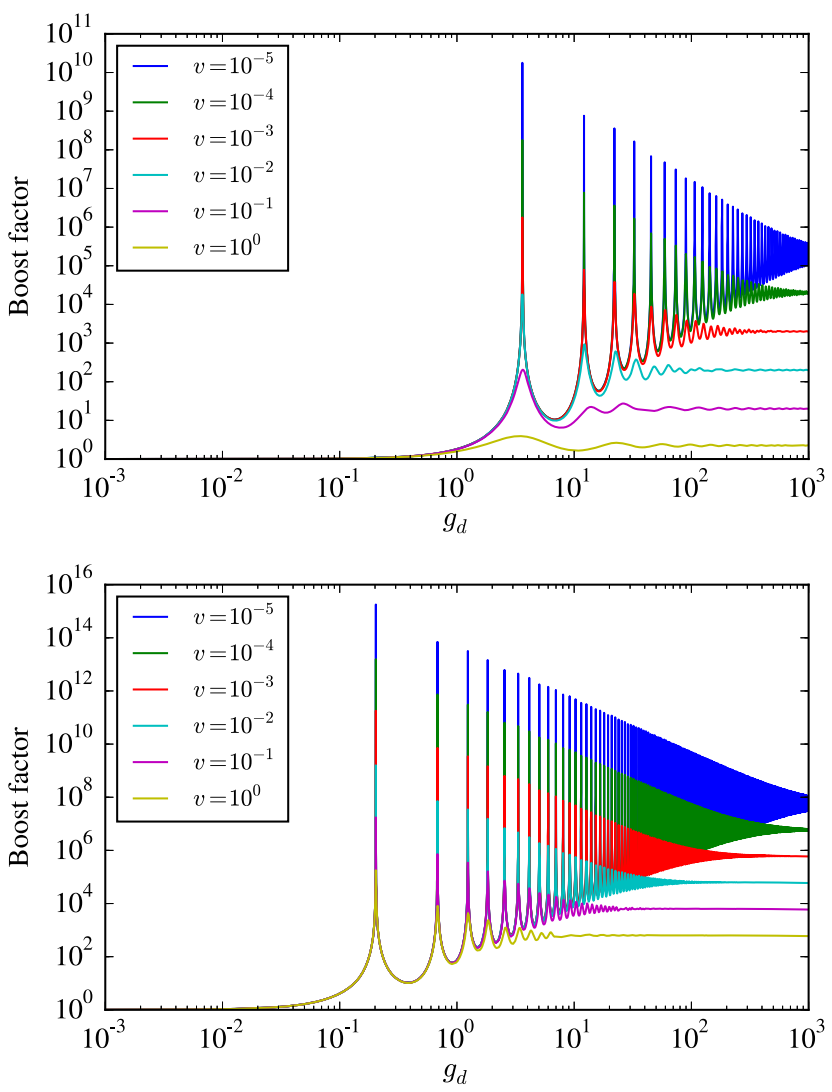

FIG. 3 (color online). Sommerfeld enhancement factor for $\ell=$ 0 due to the potential in Eq. (11) for two extreme values of the ratio $\left(m_{\chi} / \Lambda_{\mathrm{BSM}}\right)$. Top panel: $\left(m_{\chi} / \Lambda_{\mathrm{BSM}}\right)=1.0$. Bottom panel: $\left(m_{\chi} / \Lambda_{\mathrm{BSM}}\right)=10^{-5}$. As discussed in the text, the dependence on the ratio $\left(m_{\phi} / m_{\chi}\right)$ is negligible.

overall normalization of the wave function; i.e. we put $A=1$. In the asymptotic limit $x \rightarrow \infty$, the solution just becomes a sine with an amplitude and a phase shift. We have

$$
\begin{aligned}
& \Phi_{\ell}(x) \rightarrow x^{\ell+1}, \quad x \rightarrow 0, \\
& \Phi_{\ell}(x) \rightarrow C \sin \left(x-\ell \pi / 2+\delta_{\ell}\right), \quad x \rightarrow \infty .
\end{aligned}
$$

To compute the Sommerfeld factor numerically, we use Eq. (18) to set initial conditions at $x_{\text {ini }}, 0<x_{\text {ini }}<x_{\text {cut }}$. We then evolve the wave until it has reached its asymptote in Eq. (19), and we denote this point by $x_{\text {asym }}$. This happens when the wave no longer feels the potential and, for $\ell>0$, the centrifugal barrier. The Sommerfeld factor is related to the asymptotic amplitude $C$ (through the overall normalization) by the formula [45]

$$
S_{\ell}=\frac{[(2 \ell+1) ! !]^{2}}{C^{2}}=\frac{[(2 \ell+1) ! !]^{2}}{\Phi_{\ell}^{2}\left(x_{\mathrm{asym}}\right)+\Phi_{\ell}^{\prime 2}\left(x_{\mathrm{asym}}\right)} .
$$

The last expression is obtained from Eq. (19) and is numerically convenient. Note that the equation for the boost factor does not depend on the masses but only on $\Phi_{\ell}$. 
The mass dependence in Eq. (16) enters only through the ratio $m_{\phi} / m_{\chi}$ in the factor $h(F x) e^{-F x}$. This factor is $\sim 1$ when $F x \lesssim 1$, and it is easy to show that this is the case for all values of $x$ where the potential is non-negligible, provided that $\frac{m_{\phi}}{m_{\chi}}<\left(v / g_{d}\right)^{\frac{2}{3}}$. This inequality is easily satisfied for the parameter space that we are considering. The regularization procedure introduces another possible mass dependence through Eq. (17). The previous argument applies again to the factor $h(F x) e^{-F x}$, ruling out a dependence on the $\left(m_{\phi} / m_{\chi}\right)$ ratio. So the only mass dependence will enter through the ratio $\left(m_{\chi} / \Lambda_{\mathrm{BSM}}\right)$. We have shown the boost factor in Fig. 3 for two extreme values of this ratio. Evidently, the effect of Sommerfeld enhancement can be safely neglected for all reasonable values of $g_{d}$.

\section{DARK ACOUSTIC OSCILLATIONS?}

Since our model couples dark matter to a background of dark radiation, we might worry that the $\chi-\phi$ system can undergo acoustic oscillations close to the epoch of recombination and thus distort the observed CMB spectrum (see e.g. Ref. [47] for a recent discussion). The interaction around the epoch of CMB formation is primarily Compton scattering, $\chi \phi \rightarrow \chi \phi$, and we can directly compare it to the normal Compton scattering rate of photons and electrons. The Compton cross section scales as $\sigma \propto \alpha^{2} / m^{2}$ where $m$ is the fermion mass. As long as $g_{d}^{2} \ll \alpha$ and $m_{\chi} \gg m_{e}$, the dark sector acoustic oscillations will be completely negligible and therefore cosmologically safe. This of course also means that late-time Compton scatterings can be safely ignored since they have no impact on the ability of $\chi$ to cluster gravitationally. Scaling relative to the electronphoton process, we can formulate the bound as

$$
g_{d}^{2} \ll 1.6 \times 10^{-2}\left(\frac{m_{\chi}}{\mathrm{MeV}}\right) .
$$

\section{DISCUSSION}

We have studied a model with secret sterile neutrino interactions mediated by a massless or very light pseudoscalar. The model has some of the same features as the previously studied models based on Fermi-like interactions mediated by heavy vector bosons in the sense that it provides a background potential which can block the production of sterile neutrinos and resolve the apparent inconsistency between cosmology and short baseline neutrino oscillation data.

However, the model has very different late-time phenomenology. The very low mass of the pseudoscalar makes the sterile neutrino strongly self-interacting at late times, an effect which is perfectly consistent with current cosmological data but might be used to uniquely identify the model once more precise measurements become available. To accommodate the mass bound from cosmological large scale structure [32], we need $g_{s} \gtrsim 10^{-6}$ to allow the sterile neutrinos to annihilate when they become nonrelativistic. Our analysis of the CMB suggests $N_{\text {eff }} \approx 4$, and this suggestion is amplified if we also consider the direct measurements of $H_{0}$. At $95 \%$ confidence we can rule out $N_{\text {eff }}=3.046$ when we include the H0 measurement, and this formally corresponds to an upper limit on $g_{s}$ of $g_{s} \lesssim 10^{-5}$ according to Fig. 1 . However, this bound is very dependent on the set of data we have used and might both be strengthened and weakened by including more data. We finally arrive at a combined bound on $g_{s}$ of

$$
10^{-6} \lesssim g_{s} \lesssim 10^{-5}(\mathrm{CMB}+\mathrm{H} 0)
$$

A more robust determination of $N_{\text {eff }}$ would allow the possible values for $g_{s}$ to be further confined, and a precise value of $N_{\text {eff }}>3.046$ would allow us to pinpoint a corresponding coupling strength. We also note that since the fundamental coupling strength is very low and restricted to the sterile sector in this model it is unlikely to produce observable effects on neutrino physics in general (see e.g. Ref. [20] for laboratory constraints). Considering nonstandard energy loss from the protoneutron star in SN1987a also leads to an upper bound on $g_{s}$ in the $\sim$ few $\times 10^{-5}$ range (see e.g. Ref. [48] for a discussion).

In addition to the coupling to sterile neutrinos, we hypothesize that the pseudoscalar also couples to the dark matter particle. Provided that the dark matter particle is sufficiently light, this can lead to significant effects on dark matter clustering in galaxies and clusters and possibly resolve some of the apparent discrepancies between the standard $\Lambda$ CDM model and observations [49]. These discrepancies include the "too big to fail" problem [50] and the "cusp vs core" problem (see Ref. [51] and references herein) but not the "missing satellites" problem [52] which would require a stronger coupling between neutrinos and DM.

For the model to be viable, the dark matter coupling must be sufficiently low that the pair annihilations do not transfer excess entropy to the plasma of sterile neutrinos and pseudoscalars. Conversely, the dark matter coupling must be strong enough to produce an observable effect on galactic dynamics. In Fig. 4 we show these two constraints simultaneously and include the bound from warm dark matter [53]. We are left with a viable DM candidate with a mass between few $\mathrm{keV}$ and $\sim 10 \mathrm{MeV}$ and couplings from $10^{-13}$ to $10^{-5}$. A more detailed treatment of the cusp vs core and too big to fail problems could probably constrain the dark matter further, but that is beyond the scope of this article. The type of dark matter, that we have described, is very different from the normal weakly interacting massive particle cold dark matter. However, it is entirely possible that dark matter consists of an additional sterile neutrino species with extremely suppressed mixing to the active sector. If this is the case, it cannot be produced via the usual scattering and oscillation mechanism. However, unlike an $\mathrm{MeV}$ sterile neutrino 


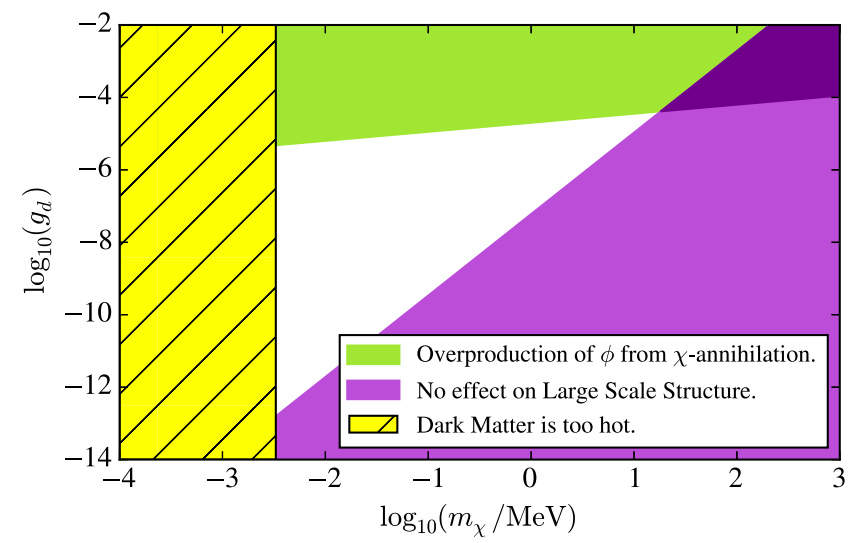

FIG. 4 (color online). Constraints in $m_{\chi}-g_{d}$ space. The green region is ruled out from Eq. (10) due to overproduction of $\phi$-particles from $\chi$-annihilations, while the purple region will have no effect on galactic dynamics, cf. Eq. (15). produced via the normal oscillation and scattering mechanism, it also remains stable on cosmological time scales. The actual production mechanism for the dark matter particle might be via direct inflaton decay at reheating or from the thermal background at very high temperature.

In summary, sterile neutrino and dark matter interactions via a light pseudoscalar seem an extremely interesting possibility for explaining a variety of different problems in cosmology and certainly merit further study.

\section{ACKNOWLEDGMENTS}

We thank the referees for comments and suggestions, which significantly contributed to improving the quality of the manuscript. M. A. acknowledges partial support from the European Union FP7 ITN INVISIBLES (Marie Curie Actions, PITN- GA-2011- 289442).
[1] J. Kopp, P. A. N. Machado, M. Maltoni, and T. Schwetz, J. High Energy Phys. 05 (2013) 050.

[2] C. Giunti, M. Laveder, Y. F. Li, and H. W. Long, Phys. Rev. D 87, 013004 (2013).

[3] K. Enqvist, K. Kainulainen, and M. J. Thomson, Nucl. Phys. B373, 498 (1992).

[4] S. Hannestad, I. Tamborra, and T. Tram, J. Cosmol. Astropart. Phys. 07 (2012) 025.

[5] N. Saviano, A. Mirizzi, O. Pisanti, P. Serpico, G. Mangano, and G. Miele, Phys. Rev. D 87, 073006 (2013).

[6] R. Cooke, M. Pettini, R. A. Jorgenson, M. T. Murphy, and C. C. Steidel, arXiv:1308.3240.

[7] P. A. R. Ade et al. (Planck Collaboration), arXiv:1502.01589.

[8] M. Archidiacono, N. Fornengo, S. Gariazzo, C. Giunti, S. Hannestad, and M. Laveder, J. Cosmol. Astropart. Phys. 06 (2014) 031.

[9] P. A. R. Ade et al., Astron. Astrophys. 571, A16 (2014).

[10] C. Dvorkin, M. Wyman, D. H. Rudd, and W. Hu, Phys. Rev. D 90, 083503 (2014).

[11] J.-F. Zhang, Y.-H. Li, and X. Zhang, Phys. Lett. B 740, 359 (2015).

[12] S. Hannestad, R. S. Hansen, and T. Tram, Phys. Rev. Lett. 112, 031802 (2014).

[13] B. Dasgupta and J. Kopp, Phys. Rev. Lett. 112, 031803 (2014).

[14] T. Bringmann, J. Hasenkamp, and J. Kersten, J. Cosmol. Astropart. Phys. 07 (2014) 042.

[15] P. Ko and Y. Tang, Phys. Lett. B 739, 62 (2014).

[16] T. Rehagen and G. B. Gelmini, J. Cosmol. Astropart. Phys. 06 (2014) 044.

[17] M. Archidiacono and S. Hannestad, J. Cosmol. Astropart. Phys. 07 (2014) 046.

[18] Y. Farzan, Phys. Rev. D 67, 073015 (2003).
[19] M. Kachelriess, R. Tomas, and J. W. F. Valle, Phys. Rev. D 62, 023004 (2000).

[20] T. Bernatowicz, J. Brannon, R. Brazzle, R. Cowsik, C. Hohenberg, and F. Podosek, Phys. Rev. Lett. 69, 2341 (1992).

[21] K. S. Babu and I. Z. Rothstein, Phys. Lett. B 275, 112 (1992).

[22] K. Enqvist, K. Kainulainen, and M. J. Thomson, Phys. Lett. B 280, 245 (1992).

[23] K. Enqvist, K. Kainulainen, and J. Maalampi, Nucl. Phys. B349, 754 (1991).

[24] B. H. J. McKellar and M. J. Thomson, Phys. Rev. D 49, 2710 (1994).

[25] G. Sigl and G. Raffelt, Nucl. Phys. B406, 423 (1993).

[26] L. Stodolsky, Phys. Rev. D 36, 2273 (1987).

[27] A. D. Dolgov, S. Pastor, J. C. Romao, and J. W. F. Valle, Nucl. Phys. B496, 24 (1997).

[28] A. Mirizzi, N. Saviano, G. Miele, and P. D. Serpico, Phys. Rev. D 86, 053009 (2012).

[29] S. Hannestad, R. S. Hansen, and T. Tram, J. Cosmol. Astropart. Phys. 04 (2013) 032.

[30] K. Kainulainen and A. Sorri, J. High Energy Phys. 02 (2002) 020.

[31] R. Barbieri and A. Dolgov, Nucl. Phys. B349, 743 (1991).

[32] A. Mirizzi, G. Mangano, O. Pisanti, and N. Saviano, Phys. Rev. D 91, 025019 (2015).

[33] S. Hannestad, J. Cosmol. Astropart. Phys. 02 (2005) 011.

[34] G. Raffelt and J. Silk, Phys. Lett. B 192, 65 (1987).

[35] F. Atrio-Barandela and S. Davidson, Phys. Rev. D 55, 5886 (1997).

[36] J. F. Beacom, N. F. Bell, and S. Dodelson, Phys. Rev. Lett. 93, 121302 (2004). 
[37] M. Cirelli and A. Strumia, J. Cosmol. Astropart. Phys. 12 (2006) 013.

[38] A. Friedland, K. M. Zurek, and S. Bashinsky, arXiv:0704.3271.

[39] A. Basboll, O. E. Bjaelde, S. Hannestad, and G. G. Raffelt, Phys. Rev. D 79, 043512 (2009).

[40] F.-Y. Cyr-Racine and K. Sigurdson, Phys. Rev. D 90, 123533 (2014).

[41] A. Lewis and S. Bridle, Phys. Rev. D 66, 103511 (2002).

[42] P. A. R. Ade et al. (Planck Collaboration), Astron. Astrophys. 571, A15 (2014).

[43] A. G. Riess, L. Macri, S. Casertano, H. Lampeitl, H. C. Ferguson, A. V. Filippenko, S. W. Jha, W. Li, and R. Chornock, Astrophys. J. 730, 119 (2011); 732, 129(E) (2011).

[44] L. Ackerman, M. R. Buckley, S. M. Carroll, and M. Kamionkowski, Phys. Rev. D 79, 023519 (2009).
[45] B. Bellazzini, M. Cliche, and P. Tanedo, Phys. Rev. D 88, 083506 (2013).

[46] P. F. Bedaque, M. I. Buchoff, and R. K. Mishra, J. High Energy Phys. 11 (2009) 046.

[47] F.-Y. Cyr-Racine, R. de Putter, A. Raccanelli, and K. Sigurdson, Phys. Rev. D 89, 063517 (2014).

[48] G. G. Raffelt, Tight Bonds Between Sterile Neutrinos and Dark Matter (Chicago University Press, Chicago, 1996), p. 664.

[49] M. Vogelsberger, J. Zavala, and A. Loeb, Mon. Not. R. Astron. Soc. 423, 3740 (2012).

[50] M. Boylan-Kolchin, J. S. Bullock, and M. Kaplinghat, Mon. Not. R. Astron. Soc. 415, L40 (2011).

[51] W. J. G. de Blok, Adv. Astron. 2010, 1 (2010).

[52] A. A. Klypin, A. V. Kravtsov, O. Valenzuela, and F. Prada, Astrophys. J. 522, 82 (1999).

[53] M. Viel, G. D. Becker, J. S. Bolton, and M. G. Haehnelt, Phys. Rev. D 88, 043502 (2013). 